\title{
The influence of resistance exercise with emphasis on specific contractions (concentric $v s$. eccentric) on muscle strength and post-exercise autonomic modulation: a randomized clinical trial
}

\author{
Mariana 0. Gois ${ }^{1}$, Fernanda A. S. Campoy ${ }^{1}$, Thâmara Alves ${ }^{1}$,
} Roseana P. Ávila ${ }^{1}$, Luiz C. M. Vanderlei ${ }^{2}$, Carlos M. Pastre ${ }^{1}$

\begin{abstract}
Background: Compared to eccentric contractions, concentric contractions result in higher cardiovascular stress. However, we do not know how these two types of contractions influence cardiac autonomic modulation during the post-exercise recovery period. Objective: to compare the effect of resistance training that is performed with concentric $v s$. eccentric emphasis on muscle strength and on post-exercise recovery which was assessed by examining heart rate variability (HRV), for the knee extensor muscle group in young healthy adults. Method: For this study, 105 men between 18 and 30 years of age were randomized into 4 groups: concentric control (CONCC), eccentric control (ECCC), concentric training (CONCT) and eccentric training (ECCT). The CONCC and ECCC groups underwent one session of resistance exercise (RE) using the knee extensor muscle group (3 sets of 1 repetition at $100 \%$ of the maximal repetition [1MR]) and the CONCT and ECCT groups performed 10 training sessions. The HRV was analyzed at baseline and across four recovery periods (T1, T2, T3 and T4). Results: The ECCT group exhibited increased muscle strength at the end of the study. Regarding cardiac autonomic modulation, the CONCC and ECCC groups exhibited increases in overall variability (SDNN and SD2) at T1 compared to baseline, and the ECCT group demonstrated increases in variables reflecting vagal modulation and the recovery process (RMSSD, SD1 and $\mathrm{HF}\left[\mathrm{ms}^{2}\right]$ ) at T1, T2 and T4 compared to baseline. Conclusions: Resistance training with emphasis on eccentric contractions promoted strength gain and an increase in cardiac vagal modulation during recovery compared to baseline.
\end{abstract}

Keywords: cardiovascular recovery; resistance training; autonomic nervous system; muscle strength; physical therapy. Clinical trial number: RBR-75scwh (recorded in Registro Brasileiro de Ensaios Clínicos - REBEC).

\section{HOW TO CITE THIS ARTICLE}

Gois MO, Campoy FAS, Alves T, Ávila RP, Vanderlei LCM, Pastre CM. The influence of resistance exercise with emphasis on specific contractions (concentric vs. eccentric) on muscle strength and post-exercise autonomic modulation: a randomized clinical trial. Braz J Phys Ther. 2014 Jan-Feb; 18(1):30-37. http://dx.doi.org/10.1590/S1413-35552012005000141

\section{Introduction}

Exercises with an emphasis on concentric or eccentric contractions have been studied in relation to outcomes such as increased strength, cardiovascular adaptation and the induction of tissue damage ${ }^{1-4}$.

Currently, most studies show that eccentric resistance exercise (RE) is more advantageous than concentric RE when considering the consumption of ATP, lactate concentrations and muscle strength output $^{1}$. On the other hand, concentric RE results in higher heart rate (HR) and blood pressure (BP) values due to increased activation of the motor units, resulting in greater cardiovascular stress when compared to eccentric contractions, which produce lower motor activation for the equivalent muscle strength output due to the stimulation of the elastic components present in the muscle-tendon units ${ }^{5-8}$.

This evidence suggests that concentric and eccentric muscle actions influence clinical and functional responses, particularly the behavior of the cardiac autonomic nervous system (ANS). Given this influence, outcomes related to post-exercise recovery should be studied. Having control of the post-exercise recovery period may reduce the risk of deleterious effects from the exercise and ensure the most appropriate exercise prescription. Studies $^{9-11}$ indicate that during the post-RE recovery

\footnotetext{
${ }^{1}$ Laboratory of Sports Physical Therapy, Department of Physical Therapy, Universidade Estadual Paulista Júlio de Mesquita Filho (UNESP), Presidente Prudente, SP, Brazil

${ }^{2}$ Stress Physiology Laboratory, Department of Physical Therapy, UNESP, Presidente Prudente, SP, Brazil

Received: 12/19/2012 Revised: 06/13/2013 Accepted: 09/27/2013
} 
process, there is a predominance of sympathetic and a reduction of parasympathetic autonomic modulations that may be associated with increased cardiovascular risk ${ }^{9-12}$.

Despite the importance of the post-exercise recovery process, our literature review did not identify any studies that evaluated cardiac autonomic modulation after exercise with emphasis on concentric and eccentric contractions. Therefore, observing the relationship between muscle strength gain and the cost of post-exercise recovery seems pertinent. Thus, the objective of this study was to compare the effect of resistance training that is performed with concentric $v s$. eccentric emphasis on muscle strength and post-exercise recovery, assessed by examining cardiac autonomic modulation, for the knee extensor muscle group in young healthy adults.

\section{Method}

\section{Study population and eligibility criteria}

For the purposes of this study, data from 105 healthy men between 18 and 30 years of age were analyzed and they were classified as physically active using the International Physical Activity Questionnaire $(\text { IPAQ })^{13}$. The objectives and procedures of the study were discussed with all of the participants, and individuals signed a consent form after agreeing to participate. All procedures were approved by the Research Ethics Committee of Universidade Estadual Paulista Júlio de Mesquita Filho (UNESP), Presidente Prudente Campus, under protocol number 20/2010.

The inclusion criteria were the absence of known cardiovascular, respiratory and/or metabolic diseases, non-alcoholic, non-smoking, no musculotendinous or osteoarticular injury episodes of the lower limbs and/ or spine in the last year and not having participated in a weight-training program for at least six months.

The participants were instructed to not consume any food and/or drinks containing caffeine for 24 hours before each session and to not dramatically alter their daily activities but, if they did so, to inform the researchers. A week before the start of the exercise protocols, testing and familiarization were carried out on the equipment and with the exercises to be performed in each group. Data collection took place in a room with controlled temperature $\left(21\right.$ to $\left.23^{\circ} \mathrm{C}\right)$ and humidity (40 to $60 \%$ ), and the records were collected between 18 and 21 hours.

\section{Allocation and groups}

The participants were randomized into four groups according to the flowchart below (Figure 1). The two control groups underwent only one session of RE. The concentric control group (CONCC) performed $\mathrm{RE}$ with a predominance of the concentric phase, and the eccentric control group (ECCC) performed $\mathrm{RE}$ with a predominance of the eccentric phase. The two training groups performed RE training, with the concentric training group (CONCT) performing training with a predominance of the concentric phase and the eccentric training group (ECCT) with a predominance of the eccentric phase. For all groups, the knee extensor muscle group was used to perform the exercises.

\section{MR Test}

Before beginning the protocol, one week was allocated to performing the $1 \mathrm{MR}$ test to measure the maximum repetition weight for the participant. The test was performed on a weight machine (Ipiranga equipment, Academia Hard line), and the maximum load, in kilograms, that each individual could achieve during knee extension was determined to ascertain training loads and those for the single RE session. The test began by using a weight equal to $50 \%$ of the body mass of the individual, and increments of $30 \%$ of this value were applied; the perceived strength of the individual was also considered. The test was completed when the volunteer reached his maximum load when executing the knee extension movement without mechanical failure. No more than five attempts to establish the maximum load were permitted. If this occurred, the test was considered invalid, and the volunteer was given the test on another day ${ }^{14}$.

If attempts at increments of $30 \%$ were higher than the volunteer could achieve, the increment was reduced to intermediate values (intervals of $5 \mathrm{~kg}$ ). If the subject reached values close to his maximum capacity and responded favorably to the slightly increased load, the load was increased using these intermediate values.

The 1MR test was performed with the volunteer's non-dominant limb because, as suggested by Gleeson et al. ${ }^{15}$, the dominant limb may be influenced by concentric and eccentric physical exertion performed during activities of daily living, enhancing the effect of muscle strength gain. The 1MR test and exercise sessions were performed on the same weight machine. During these sessions, individuals were 


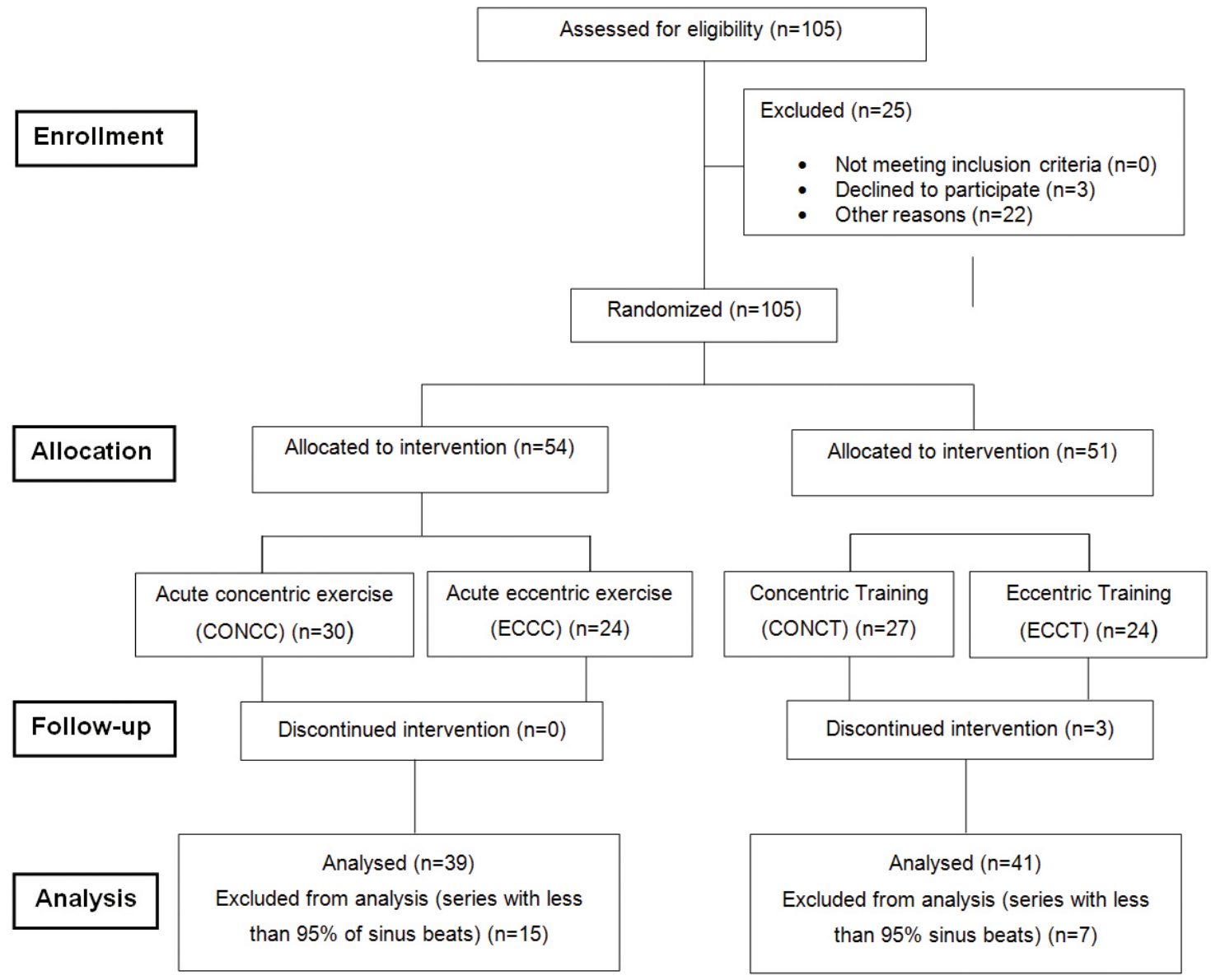

Figure 1. Flow of participants throughout the study.

secured using Velcro straps on the trunk and thighs to decrease compensatory movements.

\section{Muscle strength}

Muscle strength was evaluated using the 1MR test, as described above. The evaluation of muscle strength was performed before and after the end ( 96 hours) of both exercise protocols.

\section{Protocols}

The CONCC and ECCC groups underwent only one RE session, which corresponded to three sets of one repetition at $100 \%$ of $1 \mathrm{MR}$. The CONCT and ECCT groups underwent ten sessions of RE training, with gradual incremental adjustments.

The prescription dynamic was based on the classical form of increasing loads, respecting the volume by intensity interdependence proposed by Chiesa ${ }^{16}$ and adapted for evolution over ten sessions, performed thrice weekly, with an interval of 48 hours in between them.

Sessions 1 and 2 comprised three sets of eight repetitions at $80 \%$ of $1 \mathrm{MR}$, with 3 minutes of rest between each set; sessions 3 and 4 comprised three sets of six repetitions at $85 \%$ of $1 \mathrm{MR}$, with 2 minutes of rest between each set; sessions 5 and 6 comprised three sets of four repetitions at $90 \%$ of $1 \mathrm{MR}$, with 1 minute and 30 seconds of rest; sessions 7 and 8 comprised three sets of two repetitions at $95 \%$ of 1MR, with 40-second rest intervals, and sessions 9 and 10 comprised three sets of one repetition at $100 \%$ of $1 \mathrm{MR}$, with 20 -second rest intervals.

\section{Description of the exercise}

Each group was asked to perform a contraction of the quadriceps muscle at different contraction speeds so that one phase, concentric or eccentric, would predominate. For the CONCC and CONCT groups, 
the participants performed knee extensions beginning at $90^{\circ}$ of flexion with the non-dominant lower limb. The muscle contraction was to occur over 3 seconds, until the full range of motion (ROM) was complete. The return of the limb to its original position occurred over 1 second.

The individuals in the ECCC and ECCT groups performed the knee extensions beginning at $90^{\circ}$ of flexion over 1 second, and the eccentric contraction during flexion occurred over 3 seconds.

\section{Heart rate variability}

To capture the RR intervals (iRR), a Polar brand heart rate monitor (model S810i, Polar Electro, Finland) was used. This equipment has been previously validated ${ }^{17}$. Before the single session of control groups and final session, $10^{\text {th }}$, of training groups, participants remained at rest, breathing spontaneously and in a supine position for 20 minutes, to collect the baseline HRV. After the baseline HRV measurements, participants performed the exercise session and continued to be monitored during postexercise recovery while breathing spontaneously and in a supine position for 45 minutes.

The data obtained were initially subjected to digital filtering performed using the software from the Polar Precision Performance, version 3.0 device. Only series with more than $95 \%$ of the sinus beats were included in the study. Subsequently, manual filtering was conducted, characterized by visual inspection of the iRRs and exclusion of abnormal intervals, and series with 256 beats were analyzed by Kubios HRV software $^{18}$. Five windows were selected for analysis: baseline (pre-exercise), T1, T2, T3 and T4. The first recovery window (T1) was started 2 minutes after stopping the exercise. To form this first window, 256 beats were counted. The $\mathrm{T} 2$ recovery window began soon after the end of T1, again selecting 256 beats. The same procedure was repeated with windows T3 and $\mathrm{T} 4$, which began after the end of the 256 beats of windows $\mathrm{T} 2$ and $\mathrm{T} 3$, respectively.

\section{Linear methods}

The indices analyzed in the time domain were the SDNN, which is the standard deviation of all normal $\mathrm{iRR}$, and the RMSSD, which was calculated by taking the square root of the sum of the squared differences between the iRR on record, divided by the number of $i R R$ at a particular time minus one iRR. Both indices were expressed in milliseconds ${ }^{19}$.
In the frequency domain, the low frequency (LF [0.04 to $0.15 \mathrm{~Hz}]$ ) and high frequency (HF [0.15 to $0.4 \mathrm{~Hz}]$ ) spectral components were analyzed and expressed in $\mathrm{ms}^{2}$ and normalized units (nu). Spectral analysis was performed using Fourier's fast transform algorithm ${ }^{19}$.

\section{Nonlinear methods}

For the nonlinear methods, the standard deviation of the instantaneous beat-to-beat variability (SD1) and the standard deviation of the long-term continuous iRR (SD2) were analyzed using a Poincaré Plot ${ }^{19}$.

\section{Data analysis}

A Shapiro-Wilk test was used to determine the normality of the data distributions. The strength measurements, anthropometric variables and ages were compared between groups using a Student's $t$ test for parametric data and a Mann-Whitney test for nonparametric data.

For comparisons between contractions (concentric $v s$. eccentric), groups (control vs. training) and time (baseline vs. T1, T2, T3 and T4), repeated measures analysis of variance (ANOVA) was used. Parametric analyses were used for the SDNN, RMSSD, SD1, SD2, LF (nu), and HF (nu) variables, and nonparametric analyses were used for the $\mathrm{LF}\left(\mathrm{ms}^{2}\right)$ and $\mathrm{HF}\left(\mathrm{ms}^{2}\right)$ variables, in a two factor scheme, complemented with the SNK (Student-NewmanKeuls) and Dunn tests, respectively.

A power analysis was performed to calculate the sample size based on a pilot study. For this analysis, the primary variable (muscle strength) was expected to have a gain of $6 \mathrm{~kg}$ and a standard deviation of 7 . The power analysis thus indicates the need for 21 participants per group to achieve $80 \%$ power. All tests were performed with a significance level of $5 \%$.

\section{Results}

Table 1 presents the participants' anthropometric variables, age and strength measurements. The $1 \mathrm{MR}$ test revealed an increase in muscle strength at the final timepoint in relation to the baseline for the ECCT group.

Tables 2, 3 and 4 show the behavior of the HRV indices during the recovery period in all groups. No significant differences were observed between concentric and eccentric actions at any time analyzed. For the ECCT group, increased RMSSD, SD1 and $\mathrm{HF}\left(\mathrm{ms}^{2}\right)$ were observed at times T1, T2 and T4 
compared to baseline, showing better recovery of cardiac autonomic modulation in this group.

\section{Discussion}

The results of this study suggest a greater strength gain and better post-exercise recovery for the group that underwent training with emphasis on eccentric contraction. Regarding strength gain, there was significant difference for the ECCT group when comparing the starting $(50 \pm 11)$ and end $(55 \pm 11)$ times. Note that the findings of this study relating to strength gain corroborate those in the scientific literature. Despite the short training period (ten sessions), it is noteworthy that the gain in the training group was approximately 5\%. Hortobágyi et al. ${ }^{20}$ observed that, after 12 weeks of eccentric and concentric isokinetic training, eccentric strength increased. According to the authors, this increase in strength is related to hypertrophy of type II muscle fibers and greater neural activation.

Aagaard et al. ${ }^{21}$ also identified increased quadriceps muscle strength and increased neuromuscular activation after 14 weeks of eccentric training compared to concentric. This type of stimulation appears to promote increased neural drive and greater efficiency in fiber recruitment patterns. The increases in strength gains after eccentric training compared to concentric training seem to be well described in the literature. However, the speed of contraction may have also contributed to this finding because greater strength development can be observed at lower contraction velocities, according to Corvino et al. ${ }^{22}$.

Importantly, despite the fact that the studies cited had longer training times than those used in the present study, the ten sessions of resistance training were sufficient to show increases in muscle strength for the exercise group that emphasized eccentric contractions. Thus, this working model is proposed as

Table 1. Participant characteristics.

\begin{tabular}{ccccc} 
& \multicolumn{2}{c}{ Control } & & Training \\
& CONCC (19) & ECCC (20) & CONCT (21) & ECCT (20) \\
Age (years) & $22 \pm 3$ & $21 \pm 2$ & $20 \pm 3$ & $21 \pm 2.5$ \\
Weight (kg) & $70 \pm 11$ & $76 \pm 11$ & $70 \pm 9$ & $74 \pm 12$ \\
Height (cm) & $173 \pm 6$ & $177 \pm 9$ & $173 \pm 6$ & $176 \pm 8$ \\
BMI (kg/m²) & $23.3 \pm 3.9$ & $24.4 \pm 2.7$ & $23.4 \pm 2.8$ & $23.7 \pm 3.5$ \\
IPAQ (min/week) & $360 \pm 25$ & $362 \pm 21$ & $358 \pm 19$ & $359 \pm 24$ \\
Initial 1MR (kg) & $47 \pm 16$ & $47 \pm 13$ & $46 \pm 10$ & $50 \pm 11$ \\
Final 1MR (kg) & $45 \pm 17$ & $46 \pm 15$ & $48 \pm 11$ & $55 \pm 11 *$ \\
\hline
\end{tabular}

CONCC: concentric control; ECCC: eccentric control; CONCT: concentric training; ECCT: eccentric training. 1MR: one maximum repetition; BMI: body mass index; min: minutes. *: significantly different than the initial 1MR of the ECCT group.

Table 2. Mean and standard deviation values of the SDNN and RMSSD by group and time of analysis.

\begin{tabular}{ccccccc}
\hline & & Baseline & T1 & T2 & T3 & T4 \\
& CONCC & $48 \pm 14$ & $87 \pm 23^{*}$ & $53 \pm 15$ & $58 \pm 18$ & $62 \pm 15^{*}$ \\
SDNN (ms) & ECCC & $49 \pm 15$ & $87 \pm 26^{*} \#$ & $53 \pm 17$ & $59 \pm 16$ & $60 \pm 19$ \\
& CONCT & $56 \pm 18$ & $58 \pm 19$ & $61 \pm 22$ & $56 \pm 16$ & $57 \pm 15$ \\
& ECCT & $48 \pm 14$ & $54 \pm 12$ & $61 \pm 10^{*}$ & $59 \pm 18^{*}$ & $61 \pm 14 *$ \\
& CONCC & $40 \pm 17$ & $47 \pm 18$ & $46 \pm 16$ & $48 \pm 18$ & $46 \pm 16$ \\
& ECCC & $45 \pm 20$ & $50 \pm 21$ & $53 \pm 25$ & $51 \pm 24$ & $50 \pm 25$ \\
& CONCT & $43 \pm 17$ & $51 \pm 24 *$ & $47 \pm 20$ & $44 \pm 20$ & $43 \pm 18$ \\
& ECCT & $38 \pm 15$ & $47 \pm 16^{*}$ & $44 \pm 16 *$ & $43 \pm 15$ & $45 \pm 13 *$ \\
\hline
\end{tabular}

CONCC: concentric control; ECCC: eccentric control; CONCT: concentric training; ECCT: eccentric training. * p $<0.05$ : versus baseline; $\diamond \mathrm{p}<0.05$ : versus CONCT; \# $\mathrm{p}<0.05$ : versus ECCT. 
Table 3. Mean and standard deviation values of SD1 and SD2 by group and time of analysis.

\begin{tabular}{|c|c|c|c|c|c|c|}
\hline & & Baseline & T1 & $\mathbf{T} 2$ & T3 & $\mathbf{T} 4$ \\
\hline \multirow{4}{*}{$\mathrm{SD} 1(\mathrm{~ms})$} & CONCC & $30 \pm 12$ & $34 \pm 13$ & $36 \pm 18$ & $35 \pm 13$ & $32 \pm 11$ \\
\hline & ECCC & $33 \pm 14$ & $35 \pm 15$ & $37 \pm 18$ & $36 \pm 17$ & $35 \pm 17$ \\
\hline & CONCT & $31 \pm 12$ & $36 \pm 17 *$ & $33 \pm 14$ & $31 \pm 14$ & $30 \pm 13$ \\
\hline & ECCT & $27 \pm 11$ & $34 \pm 11 *$ & $31 \pm 11$ & $30 \pm 11 *$ & $32 \pm 9 *$ \\
\hline \multirow{4}{*}{$\mathrm{SD} 2(\mathrm{~ms})$} & CONCC & $60 \pm 19$ & $116 \pm 36^{*}$ & $65 \pm 22$ & $70 \pm 20$ & $79 \pm 21 *$ \\
\hline & $\mathrm{ECCC}$ & $55 \pm 17$ & $118 \pm 41 * \#$ & $62 \pm 18$ & $76 \pm 19 *$ & $75 \pm 23 *$ \\
\hline & CONCT & $66 \pm 20$ & $73 \pm 23$ & $72 \pm 20$ & $72 \pm 20$ & $75 \pm 19$ \\
\hline & ECCT & $65 \pm 22$ & $67 \pm 15$ & $76 \pm 18$ & $78 \pm 25$ & $80 \pm 21 *$ \\
\hline
\end{tabular}

CONCC: concentric control; ECCC: eccentric control; CONCT: concentric training; ECCT: eccentric training. $\bullet$ p $<0.05$ : versus CONCT; \# $\mathrm{p}<0.05$ : versus ECCT.

Table 4. Median, minimum, maximum, mean and standard deviation of the spectral components of HF and LF expressed in normalized units and $\mathrm{ms}^{2}$ by group and time of analysis.

\begin{tabular}{ccccccc}
\hline & & Baseline & T1 & T2 & T3 & T4 \\
& CONCC & $527(130 ; 2233)$ & $758(151 ; 2621)$ & $689(175 ; 2076)$ & $871(167 ; 1950)$ & $683(245 ; 2048)$ \\
HF $\left(\mathrm{ms}^{2}\right)$ & ECCC & $617(38 ; 5011)$ & $691(150 ; 2951)$ & $787(1050 ; 2853)$ & $666(1230 ; 3300)$ & $626(910 ; 4683)$ \\
& CONCT & $482(140 ; 2681)$ & $736(110 ; 3049)$ & $613(96 ; 2175)$ & $525(84 ; 2258)$ & $599(144 ; 2468)$ \\
& ECCT & $425(50 ; 1514)$ & $685(1360 ; 2771)^{*}$ & $685(125 ; 1577)^{*}$ & $549(138 ; 1906)^{*}$ & $807(105 ; 2299)^{*}$ \\
& CONCC & $433(209 ; 2550)$ & $908(180 ; 4747)$ & $760(183 ; 1963)$ & $756(197 ; 2117)$ & $881(4010 ; 4348)$ \\
LF $\left(\mathrm{ms}^{2}\right)$ & ECCC & $813(60 ; 3546)$ & $824(1560 ; 2494)$ & $511(196 ; 2936)$ & $969(2050 ; 5388)$ & $1061(109 ; 3181)$ \\
& CONCT & $756(18 ; 2252)$ & $816(1080 ; 2373)$ & $1035(120 ; 4221)$ & $789(217 ; 4423)$ & $920(110 ; 5625)$ \\
& ECCT & $577(2360 ; 1464)$ & $762(258 ; 2038)$ & $913(238 ; 3384)$ & $901(1960 ; 2431)$ & $1052(1950 ; 3068)$ \\
& CONCC & $53 \pm 14$ & $51 \pm 8$ & $52 \pm 13$ & $55 \pm 15$ & $59 \pm 16$ \\
LF $(\mathrm{nu})$ & ECCC & $56 \pm 18$ & $53 \pm 11$ & $49 \pm 14 *$ & $56 \pm 14$ & $62 \pm 14$ \\
& CONCT & $59 \pm 14$ & $56 \pm 14$ & $62 \pm 12$ & $62 \pm 15$ & $62 \pm 17$ \\
& ECCT & $54 \pm 14$ & $51 \pm 12$ & $56 \pm 14$ & $60 \pm 12 *$ & $57 \pm 11$ \\
HF $(\mathrm{nu})$ & EONCC & $46 \pm 14$ & $47 \pm 11$ & $47 \pm 14$ & $47 \pm 13$ & $40 \pm 16$ \\
& ECCC & $44 \pm 19$ & $47 \pm 11$ & $50 \pm 15$ & $45 \pm 14$ & $39 \pm 17$
\end{tabular}

CONCC: concentric control; ECCC: eccentric control; CONCT: concentric training; ECCT: eccentric training. HF: high frequency; LF: low frequency. ${ }^{*} \mathrm{p}<0.05$ : versus baseline.

a useful method to achieve early strength gains with a lower amount of work, which may be of interest in clinical practice, particularly when designing rehabilitation programs.

When post-exercise recovery processes are the object of study, the behavior of the parasympathetic modulation should be considered. In this regard, for the analyzed indices that reflect vagal modulation, no difference was observed between the groups (control vs. training). However, when analyzing recovery, the control group demonstrated no clear recovery response, while this response was evident in the training groups. The prevalence of a recovery response was highest in the group given eccentric training. Higher RMSSD, SD1 and $\mathrm{HF}\left(\mathrm{ms}^{2}\right)$ values were observed for the ECCT group recovery times compared to baseline.

It is difficult to compare these findings with the literature because the present study is the first to evaluate cardiac autonomic modulation during 
the recovery period after concentric and eccentric actions. The literature review only revealed studies that examine the effect of training on the behavior of HRV, i.e., the adaptation process ${ }^{2,3}$, and not its behavior as a marker of recovery. This was particularly true for studies that considered types of exercises with different dynamics.

The indices reflecting overall variability ${ }^{19,23}$ (SDNN and SD2) show an increase in the first window of recovery (T1) for the control groups. By observing the indices as a whole, it seems that such behavior is due to an interaction of sympathetic and parasympathetic components, both of which were increased at $\mathrm{T} 1$ for the CONCC and ECCC groups. Some authors ${ }^{9-11}$ have observed a predominance of sympathetic modulation when analyzing HRV indices in the frequency domain after acute RE stimulation. Heffernan et al. ${ }^{9}$ observed a decrease in the HF index (nu) and an increase in LF (nu) after $\mathrm{RE}$ and endurance exercises, but greater reductions in total power were observed only after RE. Rezk et al. ${ }^{10}$ and Andrade Lima et al. ${ }^{11}$ also observed a predominance of sympathetic modulation and a reduced parasympathetic modulation after global upper limb (MMSS) and trunk RE, respectively, performed at different intensities.

It should be noted that the aforementioned studies do not evaluate the two types of contractions in isolation, which makes further comparison impossible. They also differ from the present study in relation to the number of groups of muscles involved, exercise intensity and volume of work, factors that directly influence the behavior of cardiac autonomic modulation after exercise.

In summary, when observing cardiac autonomic behavior during the post-exercise recovery process, groups that underwent only one exercise session showed increased overall variability in the first window of recovery, and the groups that underwent training, especially the ECCT group, showed better values on indices that reflect vagal modulation (RMSSD, SD1 and HF) in the recovery windows when compared to baseline. It is therefore suggested that eccentric training leads to positive adaptation in cardiac vagal control and offers better recovery.

One limitation of this study that should be noted is the posture of the participants when the heartbeats were recorded. At both the baseline and recovery times, participants were in a supine position when the heartbeats were recorded, which did not permit the analysis of immediate cardiac autonomic modulation after exercise to evaluate vagal reentry because the exercise was performed in a seated position. The findings of this study suggest that the analysis of post-exercise recovery periods based on HRV indices should be considered, especially those that reflect parasympathetic activity. Although there are no differences between contraction types, an exploration of different stresses or pathological conditions may provide interesting findings and therefore reveal risks or safety issues for conducting workouts of any type.

\section{Conclusion}

In conclusion, the findings of this study suggest that resistance training performed with an emphasis on eccentric contractions promoted strength gains and increases in cardiac vagal modulation during the recovery process compared to the baseline condition and that there are no differences in HRV indices between contraction types across the times analyzed.

\section{Acknowledgements}

The authors would like to thank the Foundation for Research Support of the State of São Paulo (Fundação de Amparo à Pesquisa do Estado de São Paulo - FAPESP: 2010/09687-0), National Council for Scientific and Technological Development (Conselho Nacional de Desenvolvimento Científico e Tecnológico - CNPq: 476109/2010-8) for their financial support.

\section{References}

1. Roig M, Shadgan B, Reid WD. Eccentric exercise in patients with chronic health conditions: a systematic review. Physiother Can. 2008;60:146-160. http://dx.doi. org/10.3138/physio.60.2.146

2. Cooke WH, Carter JR. Strength training does not affect vagal-cardiac control or cardiovagal baroreflex sensitivity in young healthy subjects. Eur J Appl Physiol. 2003;93:719-25. http://dx.doi.org/10.1007/ s00421-004-1243-x

3. Melo RC, Quitério RJ, Takahashi ACM, Silva E, Martins LEB, Catai AM. High eccentric strength training reduces heart rate variability in healthy older men. $\mathrm{Br} \mathrm{J}$ Sports Med. 2008;42:59-63. http://dx.doi.org/10.1136/ bjsm.2007.035246

4. Seger JY, Thorstensson A. Effects of eccentric versus concentric training on thigh muscle strength and EMG. Int J Sports Med. 2005;26:45-52. http://dx.doi. org/10.1055/s-2004-817892

5. Overend TJ, Versteegh TH, Thompson E, Birmingham TB, Vandervoort AA. Cardiovascular stress associated with concentric and eccentric isokinetic exercise in young 
and older. J Gerontol A Biol Sci Med Sci. 2000;55(4):17782. http://dx.doi.org/10.1093/gerona/55.4.B177

6. Thompson E, Versteegh TH, Overend TJ, Birmingham TB, Vandervoort AA. Cardiovascular responses to submaximal concentric and eccentric isokinetic exercise in older men. J Aging Physl Act. 1999;7:20-31.

7. Okamoto TB, Masuhara M, Ikuta K. Cardiovascular responses induced during high-intensity eccentric and concentric isokinetic muscle contraction in healthy young adults. Clin Physiol Funct Imaging. 2006;26:39-44. http:// dx.doi.org/10.1111/j.1475-097X.2005.00651.x

8. Vallejo AF, Schroeder ET, Zheng L, Jensky NE, Sattler FR. Cardiopulmonary responses to eccentric and concentric resistance exercise in older adults. Age Ageing. 2006;35:291-7. http://dx.doi.org/10.1093/ageing/afj082

9. Heffernan KS, Kelly EE, Coliier SR, Fernhall B. Cardiac autonomic modulation during recovery from acute endurance and resistance exercise. Eur J Cardiovasc Prev Rehabil. 2006;13(1):80-6. PMid:16449868.

10. Rezk CC, Marrache RCB, Tinucci T. Post-resistance exercise hypotension, hemodynamics, and heart rate variability: influence of exercise intensity. Eur J Appl Physiol. 2006; 98:105-12. http://dx.doi.org/10.1007/ s00421-006-0257-y

11. Andrade Lima AHR, Forjaz CLM, Silva GQM, Menêses AL, Rofrigues Silva AJM, Ritti-Dias RM. Efeito agudo da intensidade do exercício de força na modulação autonômica cardíaca pós-exercício. Arq Bras Cardiol. 2011;96(6):498-503. http://dx.doi.org/10.1590/ S0066-782X2011005000043

12. Heffernan KS, Sosnoff JJ, Jae SY, Gates GJ, Fernhall B. Acute resistance exercise reduces heart rate complexity and increases QTc interval. Int J Sports Med. 2008;29(4):28993. http://dx.doi.org/10.1055/s-2007-965363

13. Pardini R, Matsudo S, Araújo T, Matsudo V, Andrade E, Braggion $\mathrm{G}$, et al. Validação do questionário internacional de nível de atividade física (IPAQ)- versão 6: estudo piloto em adultos jovens brasileiros. Rev Bras Ciên Mov. 2001;9(3):45-51.

14. Brown LE, Weir JP. Asep procedures recommendation I: accurate assessment of muscular strength and power. $\mathrm{J}$ Exerc Physiol Online. 2001;4(3):1-21.

15. Gleeson N, Eston R, Marginson V. Effects of prior concentric training on eccentric exercise induced muscle damage. Br J Sports Med. 2003;37:119-25. http://dx.doi. org/10.1136/bjsm.37.2.119

16. Chiesa LC. La Musculación Racional: Bases para um entrenamiento organizado. Barcelona: Editorial Paidotribo; 2007.
17. Vanderlei LCM, Silva RA, Pastre CM, Azevedo FM, Godoy FM. Comparison of the Polar S810i monitor and the ECG for the analysis of heart rate variability in the time and frequency domains. Braz J Med Biol Res. 2008;41(10):854-9. http://dx.doi.org/10.1590/ S0100-879X2008005000039

18. Niskanen JP, Tarvainen MP, Ranta-Aho PO, Karjalainen PA. Software for advanced HRV analysis. Comput Methods Programs Biomed. 2004;76(1):73-8. http:// dx.doi.org/10.1016/j.cmpb.2004.03.004

19. Vanderlei LCM, Pastre CM, Hoshi RA, Carvalho TD, Godoy MF. Noções básicas de variabilidade de frequência cardíaca e sua aplicabilidade clínica. Rev Bras Cir Cardiovasc. 2009;24(2):205-17. http://dx.doi.org/10.1590/ S0102-76382009000200018

20. Hortobágyi T, Hill JP, Houmard JA, Fraser DD, Lambert NJ, Israel RG. Adaptative responses to muscle lengthening and shortening in humans. J Appl Physiol. 1996;80(3):765-72. PMid:8964735.

21. Aagaard P, Simonsen EB, Andersen JL, Magnusson SP, Halkjaer-Kristensen J, Dyhre-Poulsen P. Neural inhibition during maximal eccentric and concentric quadriceps contraction: effects of resistance training. J Appl Physiol. 2000;89:2249-57. PMid:11090575. http:// dx.doi.org/10.1097/00005768-199805001-01178

22. Corvino RB, Caputo F, Oliveira AC, Greco CC, Denadai BS. Taxa de desenvolvimento de força em diferentes velocidades de contrações musculares. Rev Bras Med Esporte. 2009;15(6):428-31. http://dx.doi.org/10.1590/ S1517-86922009000700005

23. Task Force of the European Society of Cardiology of the North American Society of Pacing Electrophysiology. Heart rate variability standards of measurement, physiological interpretation, and clinical use. Circulation. 1996;93(5):1043-65. http://dx.doi.org/10.1161/01. CIR.93.5.1043

\section{Correspondence}

\section{Carlos Marcelo Pastre}

Univ Estadual Paulista (UNESP)

Departamento de Fisioterapia

Laboratório de Fisioterapia Desportiva (LAFIDE)

Rua Roberto Simonsen, 305, Centro Educacional

CEP 19060-900, Presidente Prudente, SP, Brasil

e-mail: pastre@fct.unesp.br 